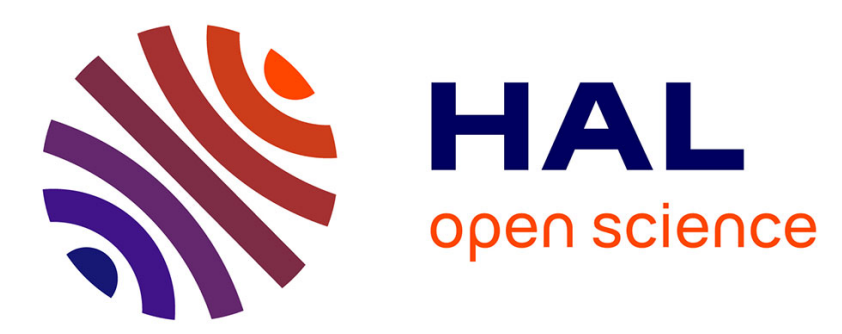

\title{
Turbulent Couette-Taylor flows with endwall effects: a numerical benchmark
}

Sébastien Poncet, Stéphane Viazzo, Adrien Aubert, Riccardo da Soghe, Cosimo Bianchini

\section{- To cite this version:}

Sébastien Poncet, Stéphane Viazzo, Adrien Aubert, Riccardo da Soghe, Cosimo Bianchini. Turbulent Couette-Taylor flows with endwall effects: a numerical benchmark. International Journal of Heat and Fluid Flow, 2013, 44, pp.229-238. 10.1016/j.ijheatfluidflow.2013.05.018 . hal-00975636

\section{HAL Id: hal-00975636 https://hal.science/hal-00975636}

Submitted on 10 Apr 2014

HAL is a multi-disciplinary open access archive for the deposit and dissemination of scientific research documents, whether they are published or not. The documents may come from teaching and research institutions in France or abroad, or from public or private research centers.
L'archive ouverte pluridisciplinaire HAL, est destinée au dépôt et à la diffusion de documents scientifiques de niveau recherche, publiés ou non, émanant des établissements d'enseignement et de recherche français ou étrangers, des laboratoires publics ou privés. 


\title{
Turbulent Couette-Taylor flows with endwall effects: a numerical benchmark
}

\author{
Sébastien Poncet,* Stéphane Viazzo, and Adrien Aubert \\ Laboratoire M2P2, UMR 7340 CNRS - Aix-Marseille Université \\ IMT La Jetée, Technopôle Château-Gombert, 38 rue F. Joliot-Curie, \\ 13451 Marseille cédex 20 - FRANCE - Fax. +0033(0)491118502 \\ Riccardo Da Soghe and Cosimo Bianchini \\ Energy Engineering Department "S. Stecco", University of Florence \\ 50139, via di S. Marta 3, Florence - ITALY
}

(Dated: February 23, 2012)

The accurate prediction of fluid flow within rotating systems has a primary role for the reliability and performance of rotating machineries. The selection of a suitable model to account for the effects of turbulence on such complex flows remains an open issue in the literature. This paper reports a numerical benchmark of different approaches available within commercial CFD solvers together with results obtained by means of in-house developed or open-source available research codes exploiting an innovative Reynolds Stress Model (RSM) closure, Large Eddy Simulation (LES) and a direct numerical simulation (DNS). The predictions are compared to the experimental data of Burin et al. [5] in an original enclosed Couette-Taylor apparatus with endcap rings. The results are discussed in details for both the mean and turbulent fields. A particular attention has been turned to the scaling of the turbulent angular momentum $G$ with the Reynolds number $R e$. By DNS, $G$ is found to be proportional to $R e^{\alpha}$, the exponent $\alpha=1.9$ being constant in our case for the whole range of Reynolds numbers. Most of the approaches predict quite well the good trends apart from the k- $\omega$ SST model, which provides relatively poor agreement with the experiments even for the mean tangential velocity profile. Even though no approach appears to be fully satisfactory, the innovative RSM closure offers the best overall agreement.

Keywords: Couette-Taylor flow ; Direct Numerical Simulation ; Large Eddy 
Simulation ; turbulence modelling.

* poncet@13m.univ-mrs.fr 


\section{INTRODUCTION}

The present investigation is concerned with the numerical modeling of fluid flow in an enclosed Couette-Taylor system. The turbulent flow is confined between two coaxial cylinders, with an inner rotating cylinder and an outer stationary one. This kind of Couette-Taylor flows is of great importance, since they found many applications in process engineering (dynamic membrane filtration, rheology, UV disinfection), in astrophysics (accretion disks) and most of all in turbomachineries for bearings, asynchronous motor with axial ventilation, rotating heat exchangers or gas turbine engines among others. In rotating machineries, a better knowledge of the fluid flow is required to better predict the heat transfer distribution and thus to optimize the performances of the device.

Up to now, only very few works have been dedicated to real industrial flows, which imply very high rotation rates and narrow gap cavity. The difficulty to perform accurate measurements in such clearances and especially in the thin boundary layers along the cylinders is obvious and has slowed down the development of specific turbulence models. For many years, the turbulent flow regime has then been treated, in computations but also in experiments, as though the flow patterns within the cavity were axisymmetric. Treating the turbulent flow as statistically stationary implicitly assumed that the coherent structures present in the transitional modes disappeared or that their effect when averaged out was accounted for in the model without explicit treatment. Owen [21] first suggested that the flow within such cavity might not be steady. In the case of rotating disk systems with throughflow, the RANS model he used failed to converge for a certain range of parameters and he speculated that some form of organized large-scale structure may have been present in the flow. These three-dimensional patterns might be responsible for some discrepancies between experiments and axisymmetric computations. Even if three-dimensional calculations may be required in some specific configurations, their highly expensive computational cost makes the selection of a suitable turbulence model for the study of rotating cavity flows still pertinent. We can cite, among others, the work of Bazilevs and Akkerman [2], who presented an application of the residual-based variational multiscale turbulence modeling methodology to the computation of turbulent Couette-Taylor flow at high Reynolds number. Its formulation globally conserves angular momentum, a feature that is felt to be important for flows dominated by rotation, and that is not shared by standard stabilized formulations of fluid flow. We can 
cite also the DNS results of Dong [6] for turbulent Couette-Taylor flows between counterrotating cylinders.

The aim of the present work is then to perform a numerical benchmarking of turbulence modellings and Large Eddy Simulations (LES) against three-dimensional direct numerical simulation (DNS) results and experimental data. The geometrical configuration corresponds to the Couette-Taylor apparatus developed by Burin et al. [5], who offered reliable velocity measurements in a rather complex geometry. Enclosed Couette-Taylor systems have been widely considered since one of the pioneering work by Wendt [30] because they offer an academic and relatively simple geometry to investigate the influence of rotation on turbulence for fundamental purposes or applications in astrophysics. When the inner cylinder is rotating while the outer one is at rest, the base flow depends mainly on three control parameters:

- the rotational Reynolds number of the inner cylinder based on the gap width $R e=$ $\Omega R_{1}\left(R_{2}-R_{1}\right) / \nu$

- the aspect ratio of the cavity $\Gamma=H /\left[2\left(R_{2}-R_{1}\right)\right]$ and,

- its radius ratio $\eta=R_{1} / R_{2}$,

where $R_{1}$ and $R_{2}$ are the radii of the inner and outer cylinders respectively and $H$ is the cavity height. Biage and Campos [4] proposed a classification of the systems depending on the value of their radius ratio $\eta$ as follows: narrow-gap cavities for $\eta>0.67$, middle-gap cavities for $0.33<\eta<0.67$ and wide-gap cavities for $\eta<0.33$. Most of the experiments up to now including the seminal one of Taylor [26] have considered cavities with an aspect ratio larger than 100. Experiments with small aspect ratios $\Gamma<10$ are less common. Kageyama et al. [14] considered both experimentally and numerically the flow in a small aspect ratio Couette-Taylor apparatus for $\eta=0.256$ and $\Gamma=0.45$. For such parameters, the vertical boundaries have a dynamical role on the secondary flow, that is why these authors proposed a novel approach to increase control over the velocity profile by increasing the number of endcap boundary conditions. Thus, they designed a new system with multiple independent endcap rings (up to 5) rotating at different speeds. Compared to classical Couette-Taylor systems, these rings allow for a reduction of the Ekman circulation in order to create a Couette-like velocity profile: $V_{\theta} \propto 1 / r^{2}$. Ji et al. [13] developed a similar Couette-Taylor 
apparatus characterized by a low aspect ratio $\Gamma=1.06$ and a low radius ratio $\eta=0.35$ with two independent end-rings between the inner and outer cylinder. Burin et al. [5] reported velocity fluctuations and the fluctuation-driven radial transport of angular momentum in the same apparatus. Fluctuation levels and the mean specific angular momentum were found to be roughly constant over radius, in accordance with previous studies featuring narrower gaps.

Couette-Taylor flows as well as Rayleigh-Bénard convection are used also as canonical flows to analyse the transport properties in closed turbulent systems with a strong analogy between them [7]. Besides their primary concern with astrophysical flows, the main goal is to understand the interplay between the boundary layers and the bulk flow. By torque measurements, Wendt [30] proposed some correlations for the dimensionless torque per unit length $G$ for $\eta=0.68,0.85,0.935$ :

$$
\begin{array}{rlrl}
G & =1.45 \frac{\eta^{3 / 2}}{(1-\eta)^{7 / 4}} R e^{1.5} & & 400<R e<10^{4} \\
G=0.23 \frac{\eta^{3 / 2}}{(1-\eta)^{7 / 4}} R e^{1.7} & & 10^{4}<R e<10^{5}
\end{array}
$$

Other experiments indicated the existence of two scaling regimes, one for small Re, where the exponent, denoted $\alpha$ in the following, is 1.5 and one for larger $R e$, where $\alpha$ remains in the range $[1.7-1.8][26,30]$. From their torque measurements, Tong et al. [27] found indeed that $G \propto R e^{1.8}$ for $4 \times 10^{4}<R e<4 \times 10^{5}$ and $\eta=0.448$. The experimental data of Burin et al. [5] for the turbulent angular momentum scale with the Reynolds number to the power $\alpha=1.7$, whatever the flow being laminar or turbulent. Dubrulle and Hersant [7] identified three logarithmic regimes using an analogy with turbulent Rayleigh-Bénard convection:

- Regime 1 for $400<R e<10^{4}$ :

$$
G=1.46 \frac{\eta^{3 / 2}}{(1-\eta)^{7 / 4}} R e^{3 / 2}
$$

- Regime 2 is not always observed in Taylor-Couette systems:

$$
G=2.12 \frac{\eta^{2 / 3}}{(1-\eta)^{5 / 3}} \frac{R e^{5 / 3}}{\ln \left[\eta^{2}(1-\eta) R e^{2} / 20\right]^{2 / 3}}
$$

- Regime 3 for $10^{3}<R e<10^{6}$ :

$$
G=0.5 \frac{\eta^{2}}{(1-\eta)^{3 / 2}} \frac{R e^{2}}{\ln \left[\eta^{2}(1-\eta) R e^{2} / 10^{4}\right]^{3 / 2}}
$$


On the other hand, the experiments of Lathrop et al. [15] and Lewis and Swinney [17] yielded no region of constant exponent. For $800<R e<1.23 \times 10^{6}$, these authors found indeed that $\alpha$ can vary between 1.6 and 1.86 in the turbulent regime. Eckhardt et al. [8] argued that there is no a single scaling but a superposition of different scalings: from $\alpha=1.5$ for small Re to $\alpha=2$ for larger ones. The exact value of $\alpha$ is then still debated since the pioneering work of Wendt [30].

The paper is organized as follows: the geometrical configuration and the numerical modelings are described in Sections II and III respectively. The results of the numerical benchmarking are presented and discussed in Section IV in terms of flow structures, mean and turbulent fields. Finally some conclusions and closing remarks are provided in Section V.

\section{DETAILS OF THE GEOMETRICAL CONFIGURATION AND FLOW CONTROL PARAMETERS}

The fluid is confined between two concentric cylinders of radii $R_{1}=0.071 \mathrm{~m}$ and $R_{2}=$ $0.203 \mathrm{~m}$ and height $H=0.28 \mathrm{~m}$ (Fig.1). The inner cylinder is rotating at a constant angular rate $\Omega$, while the outer cylinder is stationary. The originality of the apparatus developed by Burin et al. [5] is that each endcap is divided into two independently driven rings of equal radial extension. The inner cylinder and the inner ring rotate at $\Omega$, while the other walls are stationary. In the following, the radial and axial coordinates are normalized as: $r^{*}=\left(r-R_{1}\right) /\left(R_{2}-R_{1}\right)$ and $z^{*}=z / H$. The junction between the rings is then at $r^{*}=0.5$.

Under isothermal conditions, the main flow is governed by three flow control parameters: the rotational Reynolds number $R e$ based on half the hydraulic diameter $D_{H} / 2=R_{2}-R_{1}$, the aspect ratio of the cavity $\Gamma$ and the radius ratio $\eta$ defined as:

$R e=\frac{\Omega R_{1}\left(R_{2}-R_{1}\right)}{\nu}=5 \times 10^{4}, 10^{5}, 2 \times 10^{5}, 4 \times 10^{5} \quad \Gamma=\frac{H}{D_{H}}=1.06 \quad \eta=\frac{R_{1}}{R_{2}}=0.35$

The radius ratio remains in the range $0.33<\eta<0.67$, so that the cavity is considered as a middle gap cavity [4]. For enclosed systems characterized by a low aspect ratio $\Gamma$, such as in the experiment of Burin et al. [5], the choice of the boundary conditions on the endcap disks is primordial and can favour the development of large Ekman recirculations 


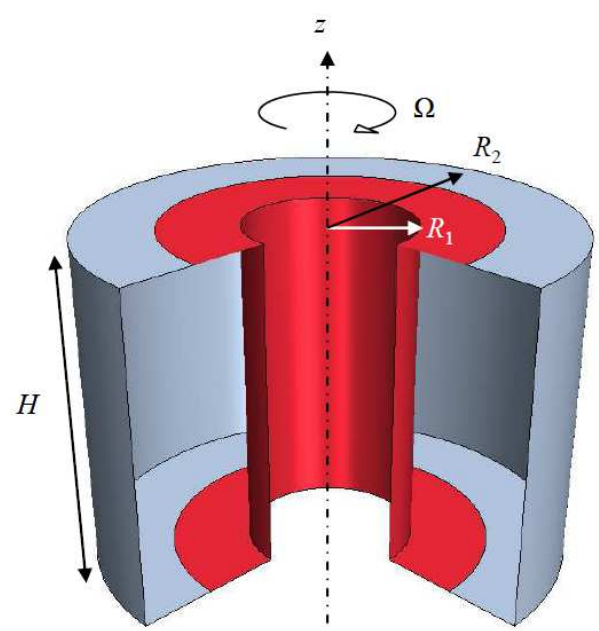

Figure 1. Geometrical configuration corresponding to the system considered by Burin et al. [5]. Red walls are rotating and grey ones are stationary.

in the meridian plane $(r, z)$. Depending on the rotation rates of the rings, the flow can be either in solid body rotation (constant angular velocity $V_{\theta} / r$ of the fluid) or present some characteristics of quasi-keplerian flows $\left(V_{\theta} / r \propto r^{-3 / 2}\right)$ or of pure Couette flows $\left(V_{\theta} / r \propto r^{-2}\right)$.

\section{NUMERICAL MODELLING}

The present flow configuration presents several complexities (high rotation rate, confinement effect and singularities in the boundary conditions among others), which are very challenging for numerical approaches. Different numerical approaches including DNS, RANS and LES models either developed at the M2P2 laboratory or at the University of Florence or available within commercial CFD codes have been compared against the velocity measurements of [5]. A brief description of the various numerical methods employed is given in the following.

\section{A. Direct numerical simulation (DNS)}

The incompressible fluid motion is governed by the three-dimensional Navier Stokes equations written in primitive variables for cylindrical coordinates $(r, \theta, z)$. No-slip boundary conditions are applied at all walls so that all the near-wall regions are explicitly computed. Conservation equations are solved using a Fourier approximation in the homogeneous tan- 
gential direction. In both non homogeneous radial and vertical directions the solutions are approximated using a fourth-order compact finite-difference scheme [1] on a structured mesh grid. The time advancement is second-order accurate and is based on the explicit Adams-Bashforth time-stepping for the convective terms and an implicit backward-Euler scheme for the viscous terms. The velocity-pressure coupling is solved using a two-step fractional scheme (predictor-corrector) reducing the problem at each time step to a set of two-dimensional Helmholtz equations.

A multidomain algorithm has also been developed in the non-periodic radial and axial directions with the aim to refine the mesh grid in some specific flow regions. The calculation domain has been then divided into four subdomains, two in each direction. The decomposition in the radial direction is to take into account the singularity in the boundary conditions between the endcap rings around $r^{*}=0.5$. The decomposition in the axial direction enables to refine the mesh grid around midplane where an intense shear layer is expected to be created by the recirculations of the secondary flow. The continuity conditions required at the interfaces of each subdomain, are computed by using the continuity influence matrix technique. This technique developed by Abide and Viazzo [1] in a Cartesian frame ensures a direct connection of $C^{1}$-type for the solution between two neighboring domains. This technique adapted here to cylindrical coordinate system allows to link the solutions in the consecutive subdomains only by one matricial calculation.

The cartesian solver has been fully validated in [3] and in [1] for the multidomain version. The numerical validation of the cylindrical solver has been done by Viazzo et al. [28] in an interdisk rotor-stator cavity in its monodomain version. The numerical details are provided in Table I. Computations have been runned on the M2P2 cluster composed of 2 Xeon quadcore $3 \mathrm{GHz}$.

\section{B. The Reynolds Stress Model of Elena \& Schiestel [9]}

The present Reynolds Stress Model (RSM) is based on one-point statistical modeling using a low Reynolds number second-order full stress transport closure sensitized to rotation effects by Elena and Schiestel [9]. This approach allows for a detailed description of near-wall turbulence and is free from any eddy viscosity hypothesis. Four additional terms account for the implicit effects of rotation on turbulence and act only when the flow is sub- 
jected to strong rotation. $\Phi^{(R)}$ is a part of the pressure-strain correlation term sensitized to the dimensionality tensor. $D^{R}$ is an inhomogeneous diffusion term, which slows down the tendency to bidimensionalization for wall bounded flows. $B$ is a homogeneous source term, which rectifies the pressure-strain correlation and which acts only in case of strong rotation. It produces spectral phase scrambling (angular dispersion). The rotation also reduces the energy transfer from large to small turbulent scales. It is modeled through an inverse flux $J$ considered as isotropic for high Reynolds numbers. The full model is given in [9].

The RSM model has been implemented in a finite-volume code using staggered grids for mean velocity components with axisymmetry hypothesis in the mean and non staggered grids for the Reynolds stress tensor. The code is unsteady elliptic. The velocity-pressure coupling is solved using the SIMPLER algorithm. In order to overcome stability problems, several stabilizing techniques are introduced in the numerical procedure. Also, the stress component equations are solved using matrix block tridiagonal solution to enhance stability using nonstaggered grids.

This version of the RSM has been fully validated in various rotating flow arrangements: rotor-stator cavity with throughflow [9, 22] and Taylor-Couette-Poiseuille flows with heat transfer [24] among others. Unsteady axisymmetric calculations using a structured mesh have been performed on the M2P2 cluster with 2 Xeon quadcore at $3 \mathrm{GHz}$. All the numerical details have been given in Table I. Note that the mesh grid has not been refined close to the junctions between the rings.

\section{The scale-adaptive simulation method within CFX}

The turbulence model considered here is the innovative $k-\omega$ SST SAS originally proposed by Menter and Egorov [19] and available within CFX 12.0. The Scale-Adaptive Simulation (SAS) is an improved URANS formulation, which allows the resolution of the turbulent spectrum in unstable flow conditions. The SAS concept is based on the introduction of the von Kármán length-scale into the turbulence scale equation. The information provided by

the von Karman length-scale allows SAS models to dynamically adjust to resolved structures in a URANS simulation, which results in a LES-like behavior in unsteady regions of the flow field. At the same time, the model provides standard RANS capabilities in stable flow regions. The governing equations of the $k-\omega$ SST SAS model differ from those of the 
SST RANS model by an additional source term in the transport equation for the turbulence eddy frequency. Further details regarding the $k-\omega$ SST SAS model are available in the literature $[19,20]$.

The calculations have been performed under unsteady isothermal conditions. The time step have been selected assuring, for each operating velocity, a RMS Courant number lower than unity. The evolving fluid is considered ideally incompressible with constant transport properties. The convective fluxes were solved using a second order upwinded scheme. Automatic wall treatment (i.e. automatic blending from low to high Reynolds treatment as a function of the local first node wall distance) have been considered. A multi-block hexahedral grid has been used. Computations have been performed on 4 cores of an Intel Xeon 3430 at $2.4 \mathrm{GHz}$. Please refer to Table I for the computational details concerning the SAS approach.

\section{Large Eddy Simulation within OpenFOAM}

OpenFOAM is an open-source CFD code composed of a set of libraries implemented to resolve continuum mechanics problems in 3D on unstructured grids [29]. A pressure based segregated solver implementing the PISO loop to solve the pressure-velocity coupling is used in transient formulation. Both the convective and diffusive flux schemes employed are based on a purely linear interpolation, time advancement is achieved by means of the implicit backward Euler scheme, guaranteeing second order accuracy both in space and in time. The time step was generally chosen to achieve a maximum Courant number inside the domain close to unity to guarantee stability and improve the convergence rate. The LES subgrid effects are evaluated by means of a one-equation model exploiting a transport equation for the modeled turbulence kinetic energy [18, 31]:

$$
\frac{\partial k}{\partial t}+\frac{\partial\left(U_{i} k\right)}{\partial x_{i}}-\frac{\partial}{\partial x_{i}}\left(\left(\nu+\frac{\nu_{S G S}}{\sigma_{k}}\right) \frac{\partial k}{\partial x_{i}}\right)=\nu_{S G S}\|S\|^{2}-\frac{c_{e} k^{3 / 2}}{\Delta} \quad, \quad \nu_{S G S}=c_{k} \sqrt{k} \Delta
$$

The LES filter width is a simple cube root of the cell volume. A multi-block hexahedral grid mesh has been used and calculations have been performed on 6 cores of an Intel Xeon 3430 at $2.4 \mathrm{GHz}$ for the case $R e=10^{5}$ (see the numerical details in Table I). 


\section{E. Large Eddy Simulation within STAR-CCM+}

Classical RANS models ( $\left.\mathrm{k}-\varepsilon, \mathrm{k}-\omega \mathrm{SST}, \mathrm{RSM}, \overline{v^{2}}-f\right)$ including both high and low-Reynolds number approaches, but also a Detached Eddy Simulation based on the Spalart-Allmaras model available within Star CCM+ 5.04 have been compared for the present test case in [11]. The LES model considered here uses a standard Smagorinsky model as subgrid scale model with a model constant $C_{s}$ equal to 0.1 offering a good compromise between the value 0.165 for homogeneous isotropic decaying turbulence and 0.07 for channel flows. The synthetic eddy method has been used to provide an initial perturbated flow field. A polyhedral unstructured mesh grid has been generated. To take into account the thin boundary layers developed along the cylinders and the rings, 12 prismatic layers are imposed on each wall on just $2 \%$ of the gap width to guarantee acceptable values for the wall coordinates (see Table I). Computations have been conducted on a HP Z400 workstation with a quadcore Intel Xeon CPU W3520 processor at 2.67 GHz.

\section{F. Computational details}

All the numerical approaches together with the corresponding computational details are summed up in Table I. Note that $N, K$ and $M$ represent the number of mesh points in the radial, tangential and axial directions respectively. Some computations have been performed considering a half cavity or only a $90^{\circ}$ sector, which is justified by the absence of large scale vortical structures as highlighted by the DNS of Fukushima et al. [10] for Re up to 12000.

\section{RESULTS}

The numerical results are compared to the velocity measurements of Burin et al. [5] in terms of mean tangential velocity and angular momentum and of the cross-correlation coefficient and the turbulent angular momentum. The DNS results provide also useful data to investigate the three-dimensional structure of the flow field. All the computed data have been averaged both in time and in the tangential direction. 


\begin{tabular}{c|c|c|c|c|c} 
modelling & time step $(s)$ & $N \times K \times M$ & geometry & wall coordinates & $\begin{array}{c}\text { time per } \\
\text { iteration }(s)\end{array}$ \\
\hline \hline DNS & $3 \times 10^{-5}$ & $152 \times 64 \times 242$ & $3 \mathrm{D}$ & $r^{+}<4$ & 17.2 \\
$z^{+}<6$ & $r^{+}<0.9$ & 70 \\
\hline LES (OpenFOAM) & $4 \times 10^{-4}$ & $200 \times 70 \times 300$ & $3 \mathrm{D}$ & $z^{+}<1.3$ & \\
\hline LES (Star CCM+) & $10^{-3}$ & $74 \times 104 \times 115$ & $30^{\circ}$ sector $)$ & $r^{+}<0.7$ & 13.4 \\
\hline RSM [9] & $10^{-2}$ & $140 \times 1 \times 280$ & $2 \mathrm{D}$ & $z^{+}<0.95$ & \\
\hline $\mathrm{k}-\omega$ SST SAS & $5 \times 10^{-5}$ & $140 \times 5 \times 280$ & axisymmetric & $z^{+}<0.06$ & 16 \\
\hline
\end{tabular}

Table I. Computational details for $R e=10^{5}$.

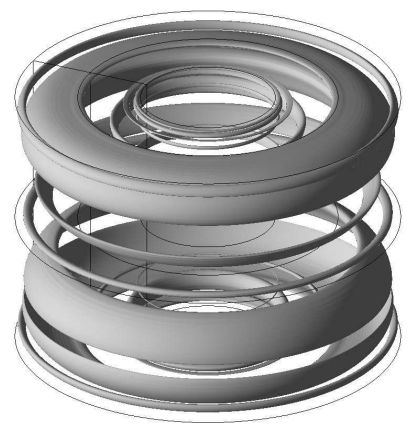

(a)

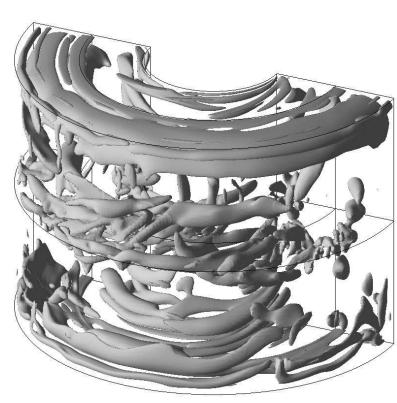

(b)

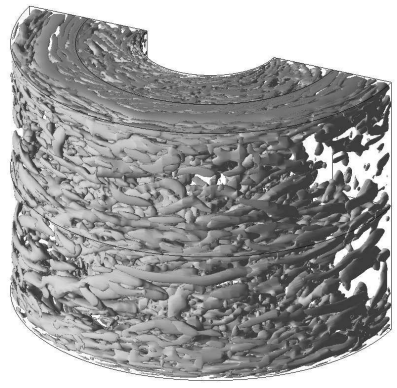

(c)

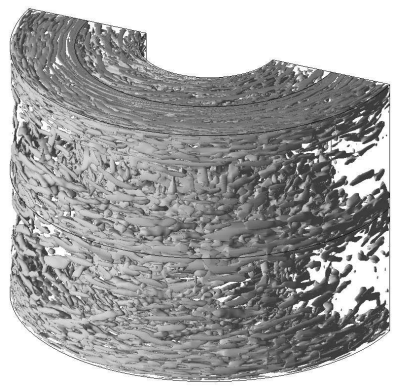

(d)

Figure 2. Iso-values of the Q-criterion obtained by DNS for (a) $R e=5 \times 10^{3}$, (b) $R e=10^{4}$, (c) $R e=5 \times 10^{4}$ and (d) $R e=10^{5}$.

\section{A. Flow structures}

The critical Reynolds number $R e$ for the transition to turbulence in such a flow depends upon a large number of factors, including the aspect ratio of the cavity, the curvature and the flow history. Nevertheless, one commonly admitted value deduced from the Reynolds 
number dependence of the mean torque at the inner cylinder is around $10^{4}[15]$. In the present case, the transition to a state of featureless turbulence appears for lower values of $R e$. At very low $R e$ values, the flow is laminar unstable with some circular rolls of different sizes propagating towards the gap $\left(R e=5 \times 10^{3}\right.$, Fig.2a). They are somewhat different from classical Taylor vortices, which would have occupied the whole cavity with a corresponding axial wave number of 2 (close to $2 \Gamma$ ). At $R e=10^{4}$ (Fig.2b), some spiral patterns aligned with the tangential direction appear along the inner rings and around the midplane $\left(z^{*}=0.5\right)$. They coexist with the circular rolls on the top and bottom stationary rings. Some evidence of a disorganized flow structure can be seen from this Q-criterion map. When one increases further the Reynolds number, the flow gets clearly turbulent at $R e=5 \times 10^{4}$ (Fig.2c) with fine scale eddies elongated in the tangential direction. Some circular rolls (not shown here) persist along the inner cylinder, with a structure similar to the toroidal vortices observed by Biage and Campos [4] for $R e=2.04 \times 10^{5}, \eta=0.38$ and $\Gamma=10$. At $R e=10^{5}$, the iso-values of the Q-criterion obtained by DNS (Fig.2d) show that the flow is clearly turbulent with even thinner turbulent structures. There is absolutely no evidence of large scale vortical structures embedded in the flow apart from large circular patterns, which appear along the inner cylinder. Turbulence is mainly concentrated at midplane $\left(z^{*}=0.5\right)$ because of the shear produced by the intense recirculation bubbles (Fig.4). Thus, the critical Reynolds number for the transition to turbulence is not significantly modified by the presence of the endcap rings compared to classical Couette-Taylor flows.

\section{B. Mean hydrodynamic field}

In such configuration, the main flow is directed tangentially due to the high rotation rate of the inner cylinder and rings. Due to centrifugal and confinement effects, a secondary flow develops in the meridional plane $(r, z)$. The results for the mean field are first qualitatively analysed considering bidimensional streamlines.

Figure 3 presents the streamline patterns obtained by DNS and the RSM for $R e=$ $5 \times 10^{4}$. In the bulk of the fluid, pressure balances the centrifugal force. The pressure being approximately uniform axially, the difference in centrifugal force between the bulk flow and the endcap rings generates a radial secondary flow in the $(r, z)$ plane. The fluid is pumped along the rotating disks and being not blocked by the fixed rings, continues to 
flow radially outward. After that, it moves axially to the center of the cavity. Then the two axial flows create an intense shear layer at midplane, where the flow is mainly radially inwards. By conservation of mass, the fluid goes back axially along the inner cylinder to the rotating rings. Thus, the two approaches predict a meridional circulation with a 2 -lobe flow structure, known as Ekman cells. Some small bubbles can be observed at the junction between the outer rings and the outer cylinder. The DNS predicts also 2 small recirculations along the inner cylinder.

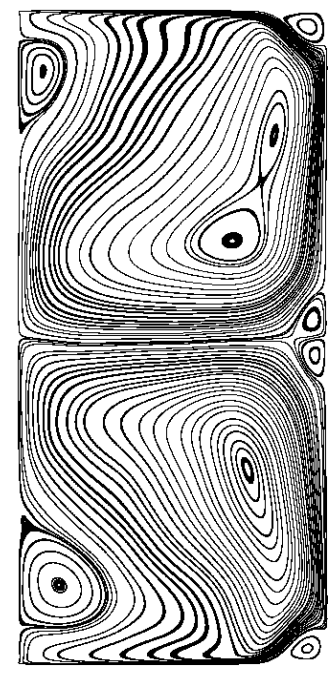

(a)

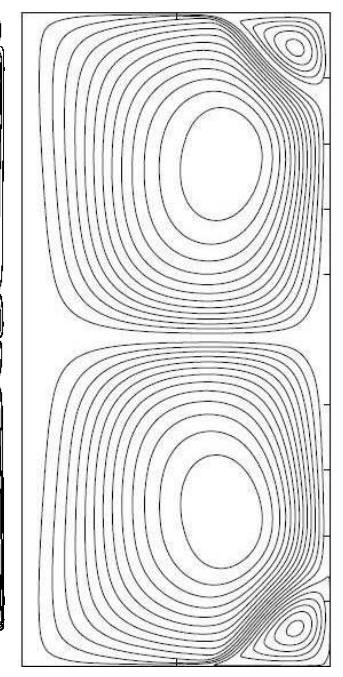

(b)

Figure 3. Mean streamline patterns obtained at $R e=5 \times 10^{4}$ by (a) DNS and (b) RSM. The rotation axis is located on the left of each subfigure.

When one increases the Reynolds number up to $R e=10^{5}$, very different patterns arise from the different computations (Fig.4). The DNS (Fig.4a), LES (Fig.4d\&e) and RSM (Fig.4b) predict a main big vortex for each of the two sides with some differences between these approaches. Looking at the streamline patterns obtained by DNS, the $k-\omega$ SST SAS (Fig.4c) and the LES of StarCCM+, asymmetry can develop at this Reynolds number. The secondary flow structure in the meridional plane gets more complex with the presence of small vortices arising along the inner cylinder or in the corners between the rings and the cylinders. 


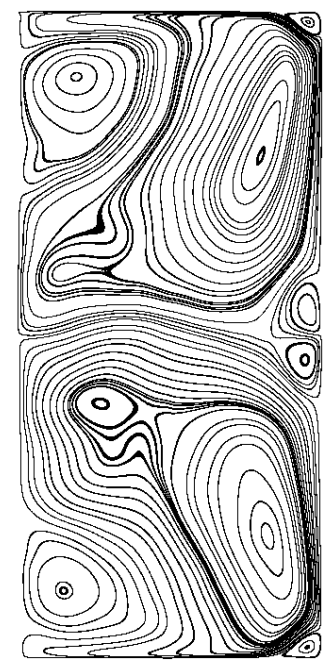

(a)

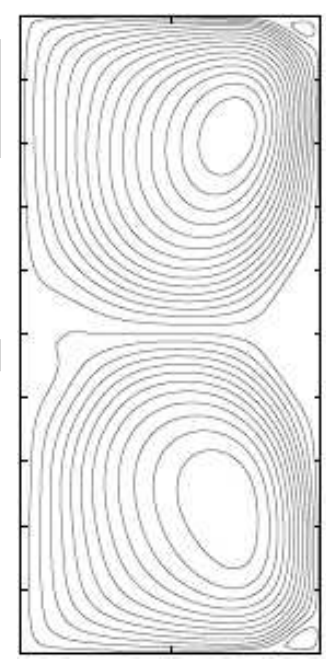

(b)

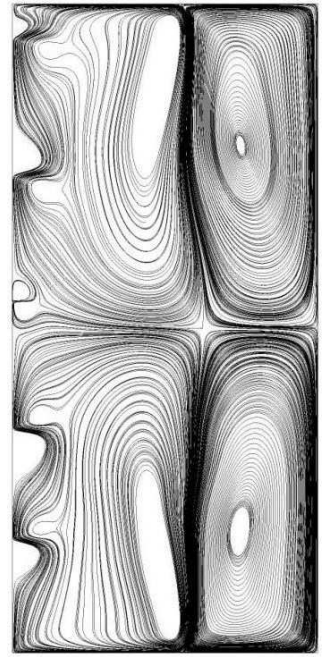

(c)

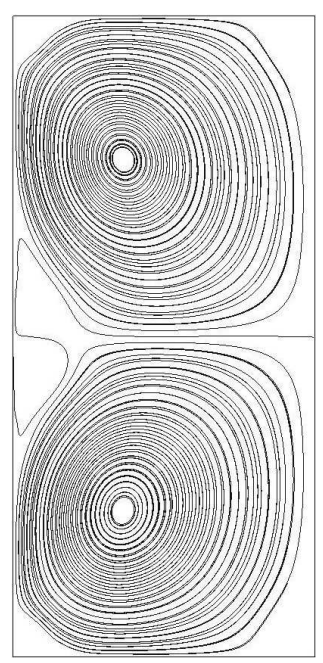

(d)

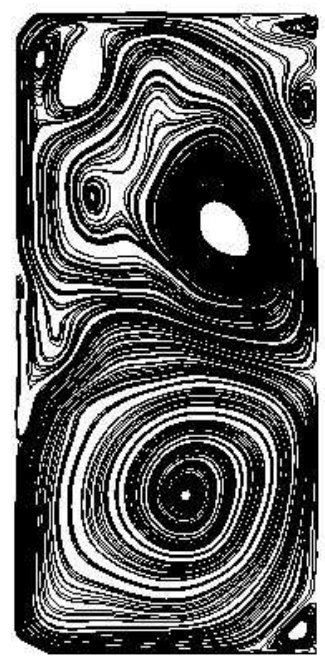

(e)

Figure 4. Mean streamline patterns obtained at $R e=10^{5}$ by (a) DNS, (b) RSM, (c) k- $\omega$ SST SAS, (d) LES (OpenFOAM) \& (e) LES (Star CCM+). The rotation axis is located on the left of each subfigure.

The k- $\omega$ SST SAS model predicts a mean velocity pattern characterized by four rotating structures at the meridional plane, which is confirmed by computations performed by other $\mathrm{k}-\omega$ models, indifferently in their unsteady or steady-state formulations and whatever the flow solver: CFX, OpenFOAM or STAR CCM+ (some results can be found in [11]). The fluid pumped along the rotating disks is deviated away from the wall almost as soon as it reattaches on the fixed rings creating a high tangential velocity zone around mid-radii (Fig.5). Such movement towards the center of the cylinder creates two counter rotating bubbles in the inner and outer zone. While the outer bubble is almost symmetric in the axial direction, the inner one is centered quite close to the rotating disk showing a quite stretched shape. Smaller recirculations attached to the inner cylinder can also be observed. These smaller vortical structures are formed close to mid-height of the cylinder and are convected towards the rings.

Figure 5 presents the radial profiles of the mean tangential velocity component $V_{\theta}^{*}=$ $V_{\theta} /\left(\Omega R_{1}\right)$ at the axial position $z^{*}=1 / 4$ for four Reynolds numbers from $R e=5 \times 10^{4}$ to $R e=4 \times 10^{5}$. At $R e=10^{5}$ for example (Fig.5b), all the numerical approaches apart from 


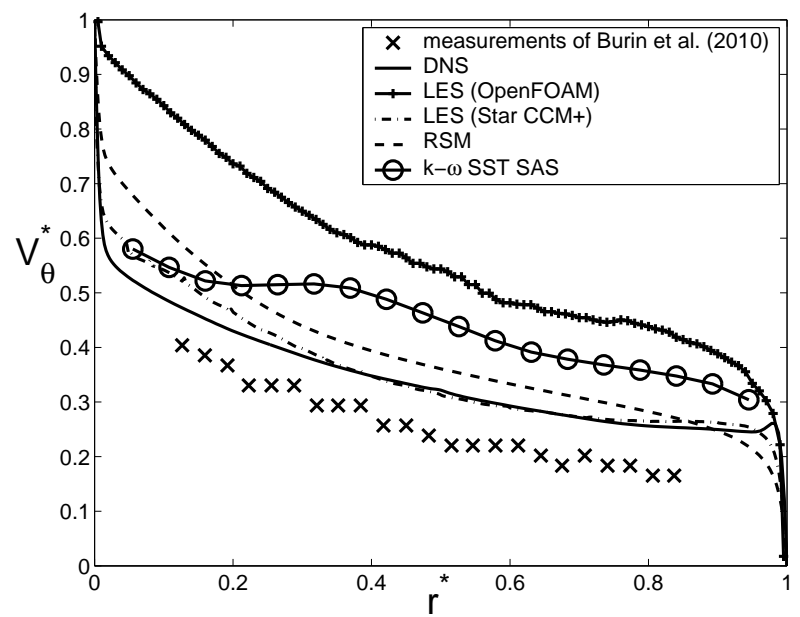

(a)

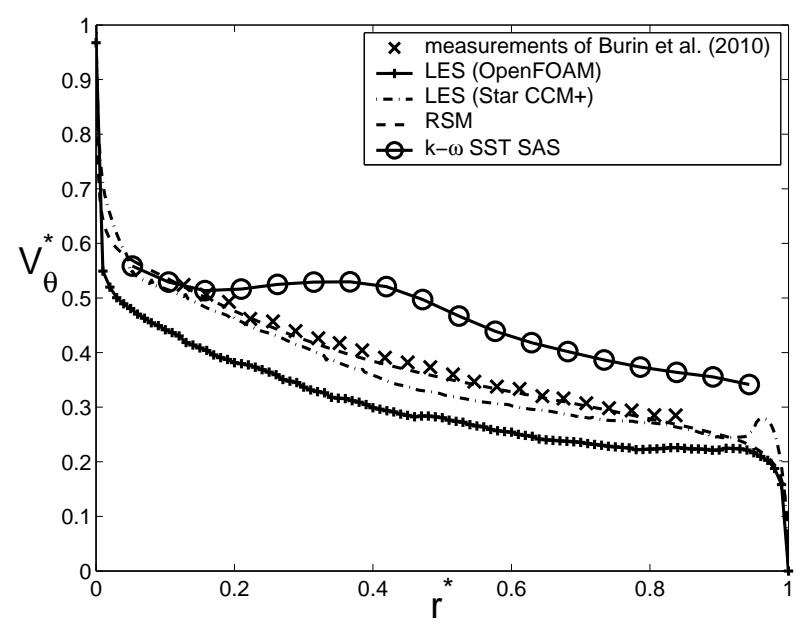

(c)

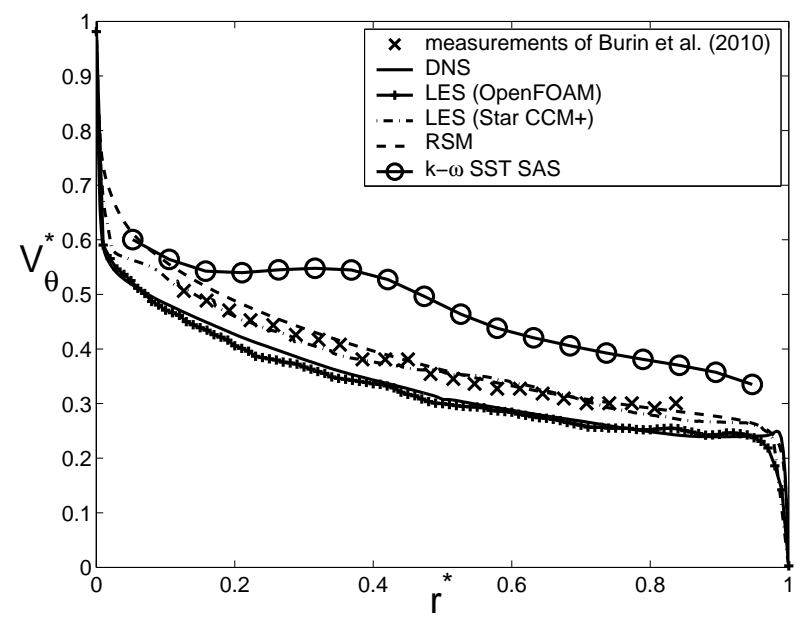

(b)

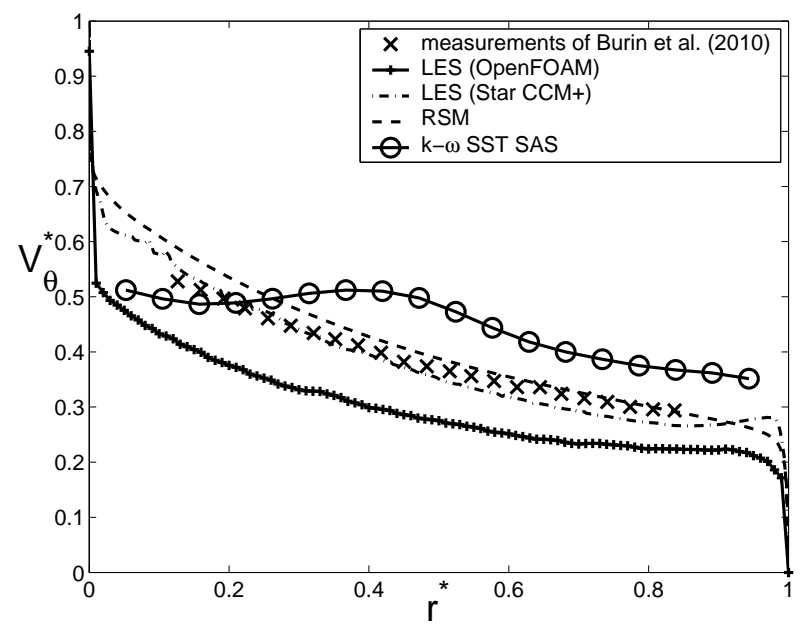

(d)

Figure 5. Radial profiles of the mean tangential velocity at $z^{*}=1 / 4$ for (a) $R e=5 \times 10^{4}$, (b) $R e=10^{5}$, (c) $R e=2 \times 10^{5}$ and (d) $R e=4 \times 10^{5}$.

the k- $\omega$ SST SAS model predict a radial profile of the mean tangential velocity divided into three areas. Two very thin boundary layers develop along the cylinders with a thickness of the order of $1 \%$ of the gap width. They are separated by a central region where the tangential velocity component scales like: $V_{\theta}^{*}(r)=0.43 \times r^{* 2}-0.78 \times r^{*}+0.59$ (from the DNS). Hollerbach and Fournier [12] pointed out that, at this Reynolds number, the shear layers developed close to the discontinuity should penetrate into the cavity and create a small discontinuity in the velocity profile. Burin et al. [5] attributed the absence of this discontinuity to an inadequate resolution of their measurements. The present data do not indicate any discontinuity in the velocity profile confirming the results of [5]. 
The k- $\omega$ SST SAS model as well as all the other k- $\omega$ SST models considered in [11] fail to predict the expected profile with a large overestimation of the mean tangential velocity within the gap (Fig.5b). While most of all the models predict $V_{\theta}^{*}\left(r^{*}=0.5\right)$ between 0.3 (LES OpenFOAM) and $0.36(\mathrm{RSM})$, the SAS model predicts $V_{\theta}^{*}(0.5) \simeq 0.48$. A local maximum is also observed around $r^{*} \simeq 0.36$ from the SAS results, which is not the case from the experiments. The unsatisfying agreement between the k- $\omega$ SST and the experimental data in terms of tangential velocity is probably related to the presence of the four rotating structures on the meridian plane. The velocity peak close to the radius $r^{*}=0.36$ on Figure 5 can be indeed justified by the tangential momentum transport operated by these inner vortices. Its behavior is similar to what obtained both experimentally and numerically by Kageyama et al. [14] for $R e \simeq 10^{6}, \eta=0.256$ and $\Gamma=0.45$. The tangential velocity profile is nearly constant over radius in their case and the authors attribute it to the reduction of the Ekman cells due to endcap ring effects. It is noteworthy that the DNS predicts a local maximum close to the outer cylinder but due to the lack of experimental data very close to the wall, no definitive conclusion can be drawn.

By comparing Figures 5a to 5d, a noticeable influence of the Reynolds number on the mean velocity profiles can be observed. For $R e \geq 10^{5}$, the mean tangential velocity slightly increases for increasing values of $R e$ but this effect remains weak. It is besides not observed from the LES (OpenFOAM) results, which underpredicts the tangential fluid velocity in that cases. The SAS model still overestimates $V_{\theta}^{*}$ in the main part of the gap. For $R e=5 \times 10^{4}$ (Fig.5a), the experimental profile is much lower, which is characteristic of much weaker turbulence levels. All the numerical approaches fail to reproduce the good profile. The DNS seems to offer the best agreement with the experiment. The overestimation of the tangential velocity of the fluid may be attributed to higher turbulence intensities and to an earlier transition to the fully turbulent regime in the numerics. Surprisingly, the LES of OpenFOAM strongly overestimates the mean velocity profile in that case, with a profile quite similar to the laminar circular Couette profile, indicating much lower turbulence levels than in the experiments or in the DNS for examples.

The same data can also be plotted in terms of the angular momentum $L_{\theta}=r V_{\theta}$ of the mean flow. Thus, Figure 6 presents the radial profiles of $L_{\theta}$ at the same axial location 


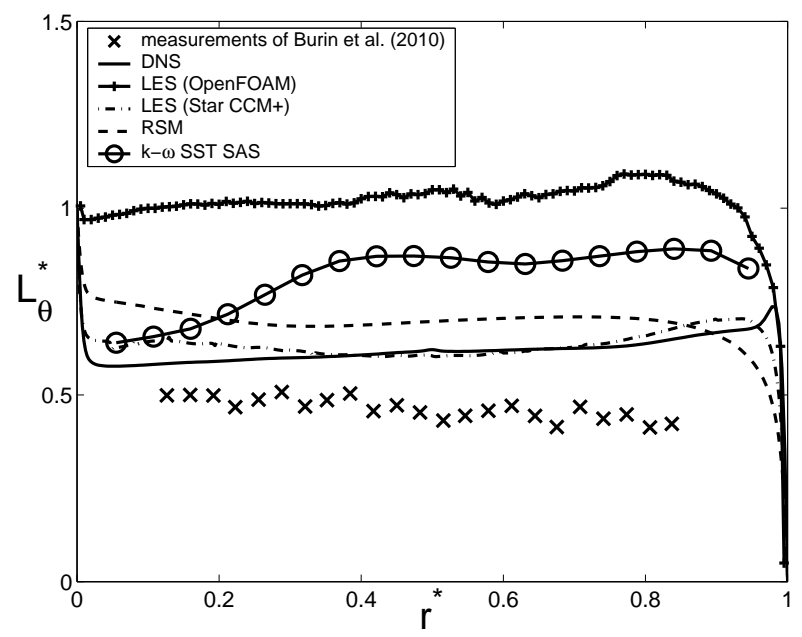

(a)

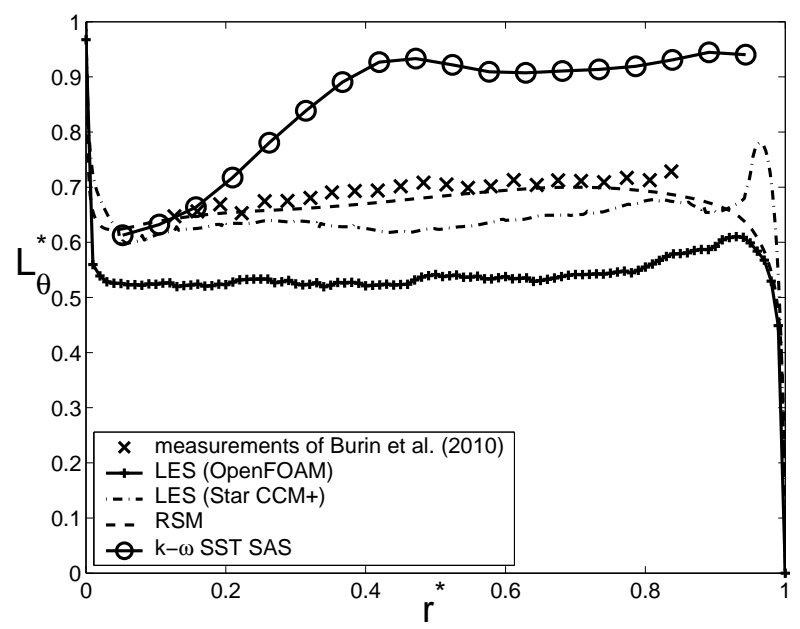

(c)

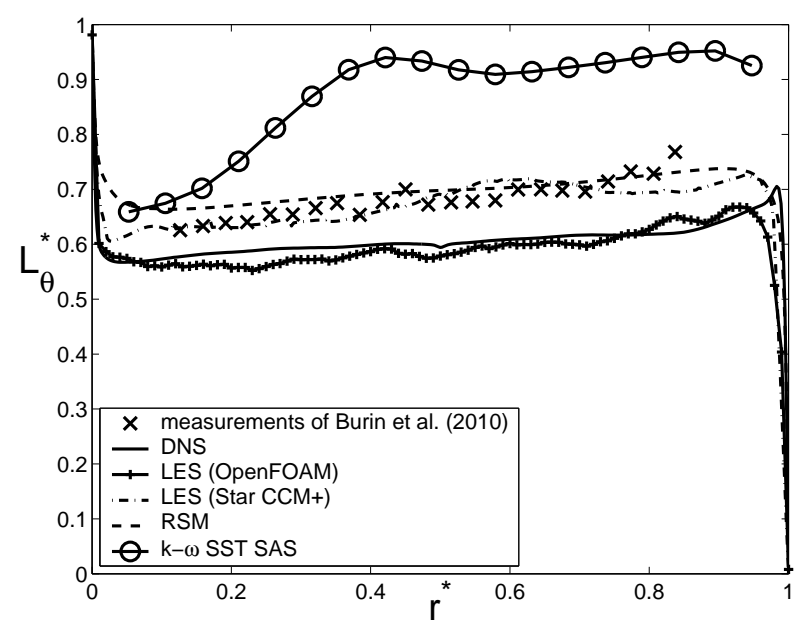

(b)

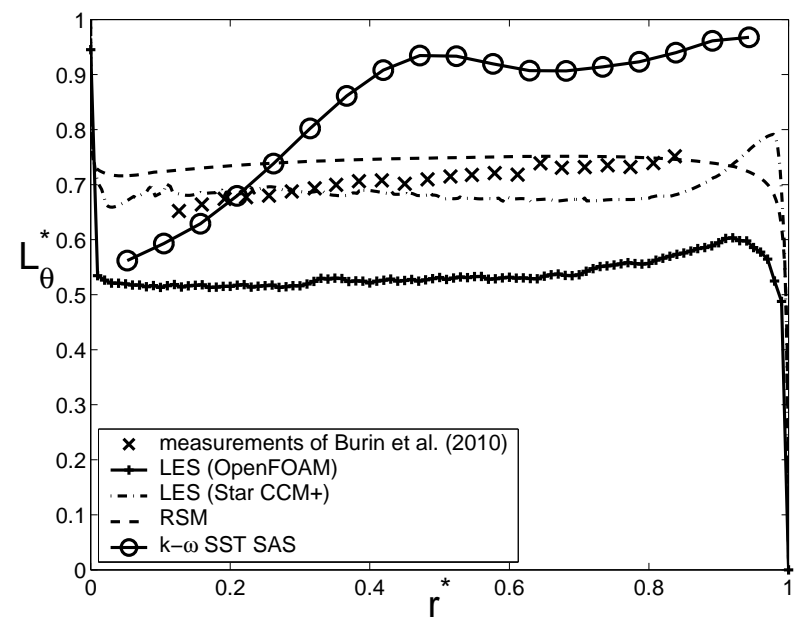

(d)

Figure 6. Radial profiles of the normalized angular momentum at $z^{*}=1 / 4$ for (a) $R e=5 \times 10^{4}$, (b) $R e=10^{5}$, (c) $R e=2 \times 10^{5}$ and (d) $R e=4 \times 10^{5}$.

$z^{*}=1 / 4$. It has been made dimensionless using the value of $L_{\theta}$ at the inner cylinder $L_{\theta}\left(R_{1}\right)=\Omega R_{1}^{2}$.

For $R e=5 \times 10^{4}$, the angular momentum is rather constant over the radius with a measured value around 0.5 , whereas a slight increase may be observed from the numerical profiles with $L_{\theta}^{*}\left(r^{*}=0.5\right) \simeq 0.62$ for the DNS. Surprisingly, the LES of OpenFOAM predicts an angular momentum, which can be slightly higher than one. For $R e \geq 10^{5}$, the influence of $R e$ on $L_{\theta}^{*}$ remains weak. Apart very close to the walls, the numerical approaches predict an angular momentum almost constant over the radius, while a slight increase with $r^{*}$ may 
be noticed from the measurements. The value at mid-gap is around 0.65 , which is much larger than the usual value 0.5 obtained in classical Couette-Taylor flows [25]. It can be explained by considering the additional torque from the inner rotating rings, which rotate at the same rate as the inner cylinder but at a larger radius. Even if most of the models predict quite well the mean tangential velocity, the variations of the angular momentum with the radius highlight larger discrepancies mainly because of the normalization. The DNS slightly underestimates the slope of the profile in the central region, where the RSM of Elena and Schiestel [9] offers the best agreement with the experimental data of Burin et al. [5]. The k- $\omega$ SST SAS model strongly overestimates the mean angular momentum in the whole gap.

\section{Turbulent statistics}

\section{Cross-correlation coefficient}

Burin et al. [5] provided also the variations with the Reynolds number of the crosscorrelation coefficient $C_{r \theta}$ defined as:

$$
C_{r \theta}=\frac{\overline{v_{\theta}^{\prime} v_{r}^{\prime}}}{\sqrt{\overline{\overline{v_{\theta}^{\prime 2}}}} \sqrt{\overline{v_{r}^{\prime 2}}}}
$$

The variation of $C_{r \theta}$ with the Reynolds number $R e$ are provided on Figure 7. Burin et al. [5] observed that $C_{r \theta}$ slightly decreases with $R e$ as the flow becomes increasingly random. As it can be seen from Figure 7, the time averaged value obtained by the different models at the monitoring point is close to $20 \%$ and does not vary so much with $R e$ in the range $\left[500,4 \times 10^{5}\right]$, in quite good agreement with [5]. For $R e=5 \times 10^{4}$, Smith and Townsend [25] found $C_{r \theta}=30-40 \%$. This value has been found using the RSM of StarCCM+ (not shown here), whereas all the other approaches predict $C_{r \theta}=0.24$ or less. The high value obtained by [25] is due, according to [5], to the presence of large scale vortical structures embedded in the flow, which has not been observed here by none of the present models.

In fact, the LES results (OpenFOAM) show that the cross-correlation coefficient strongly varies between -0.4 and 0.4 depending on the computed position in a meridian plane, as it 


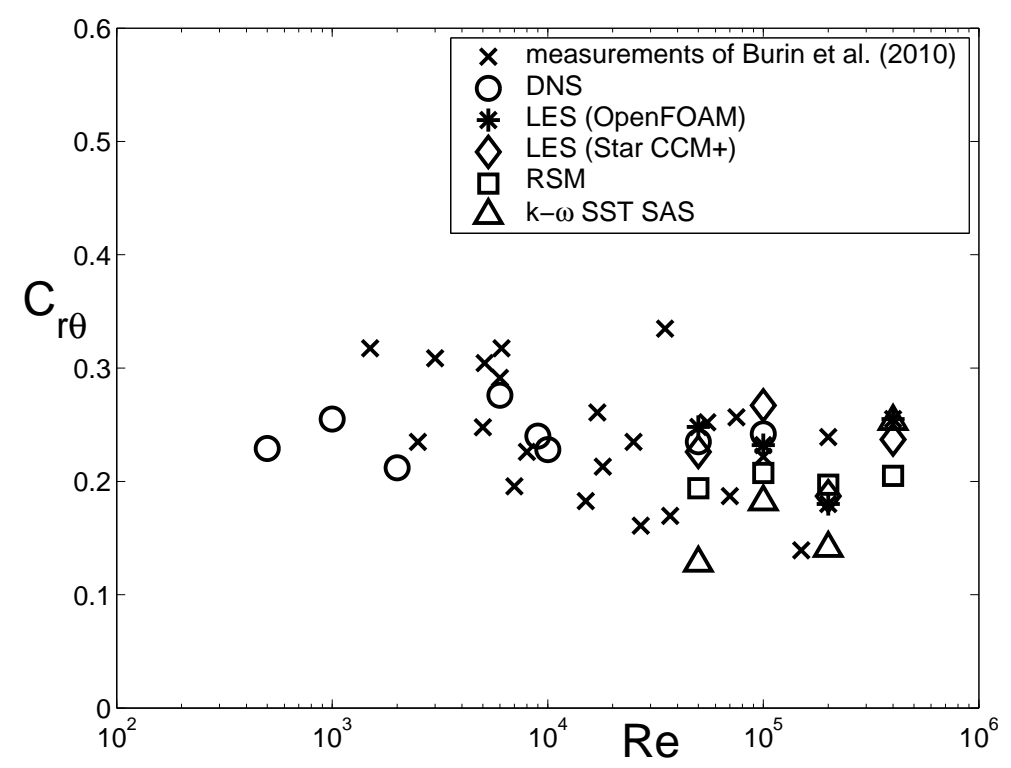

Figure 7. Variations of the cross correlation coefficient $C_{r \theta}$ with the Reynolds number $R e$ at $z^{*}=1 / 4$.

can be seen from the $C_{r \theta}$-map in Figure 8. Thus, those punctual values have to be considered carefully and might be not so representative of the time averaged cross correlation in that region.

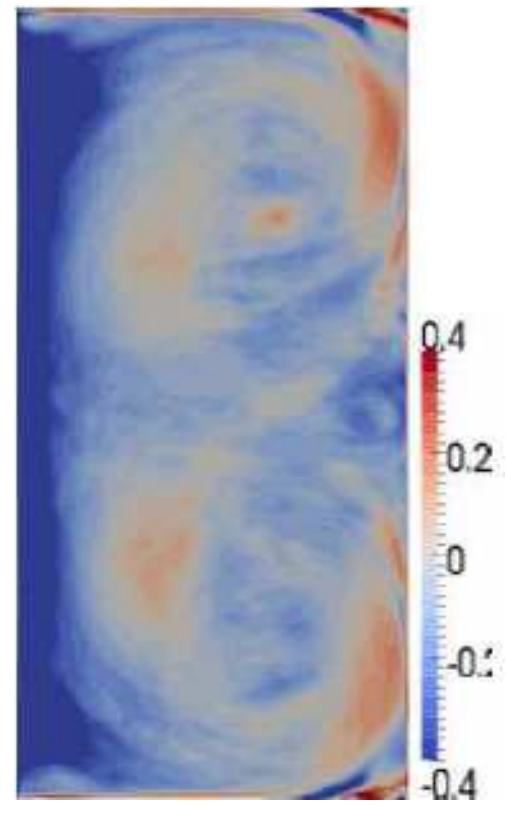

Figure 8. Map of the cross-correlation coefficient $C_{r \theta}$ in a meridian plane obtained by the LES of OpenFOAM at $R e=10^{5}$. 


\section{Transport of turbulent angular momentum}

The measured velocity correlation is discussed also in terms of a dimensionless torque per unit length $G$, in which the viscous term has been neglected as in [5]:

$$
G=\frac{2 \pi r^{2} \overline{v_{\theta}^{\prime} v_{r}^{\prime}}}{\nu^{2}}
$$

Its variation with the Reynolds number is shown in Figure 9 for all the approaches and compared to the measurements of [5]. It is also compared to the scalings proposed by Dubrulle and Hersant [7] (Eq.??). For this range of Reynolds numbers, the DNS, LES (OpenFOAM) and RSM results confirm that $G$ depends on $R e$ following an unique power law: $G \propto R e^{\alpha}$ with $\alpha=1.9$, which suggests that the transport of angular momentum may be seen as a diffusive process. This value remains slightly larger than the one $\alpha=1.7$ deduced from the measurements of Burin et al. [5] or Wendt [30]. Tong et al. [27] obtained $\alpha=1.8$ for $\eta=0.448$ and $4 \times 10^{4}<R e<4 \times 10^{5}$. It shows that only one regime is obtained here corresponding to the regime 3 of Dubrulle and Hersant [7]. Equation 5 would fit indeed quite well our numerical results with a lower radius ratio $\eta$.

Most of the models underestimate the values of $G$ compared to the predictions of Equation (5) or to the measurements of [30]. Two main reasons can be advocated in the present case. Firstly, the aspect ratio of the Burin et al.'s [5] apparatus is low compared to classical Couette-Taylor systems, which increases the influence of the endcap disks on the flow. The fact that only the inner rings are rotating with the inner cylinder may partly explain the reduced values of the turbulent angular momentum. Secondly, looking at Figure 10, one may also argue whether comparing a punctual value is an efficient strategy to verify turbulence levels when, as this is the case here, the local variability of the resolved part of $G$ is high. Figure 10 shows also how the modeled contributions are important only very close to the outer walls and are of the same order of magnitude as the resolved part in that regions. 


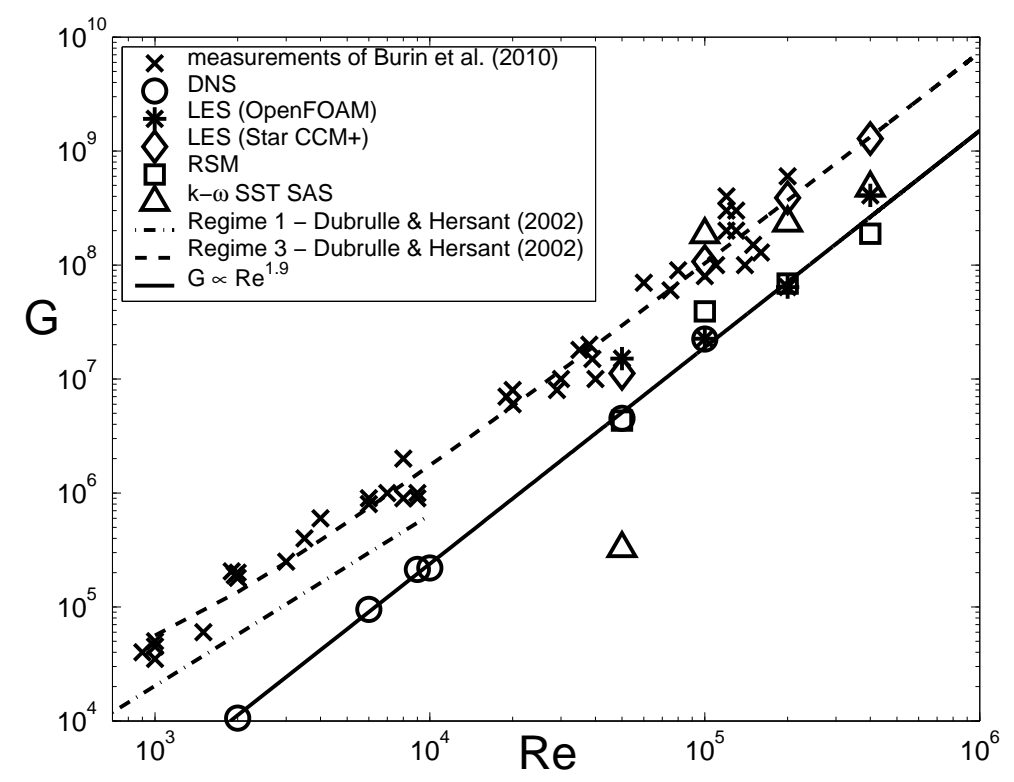

Figure 9. Variations of the turbulent angular momentum transport $G$ with the Reynolds number Re at $z^{*}=1 / 4$.

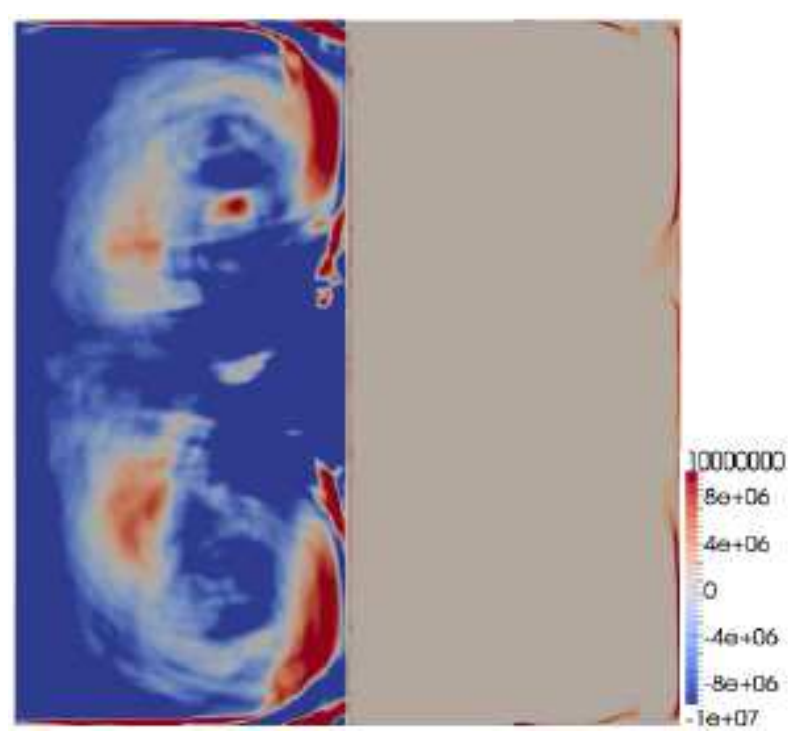

Figure 10. Map of the turbulent angular momentum $G$ (resolved and modelled parts) in a meridian plane obtained by the LES of OpenFOAM at $R e=10^{5}$.

A preliminary calculation on a 5 degree sector using the LES within OpenFOAM has been performed providing much lower values of both $C_{r \theta}$ and $G$ with at least one order of magnitude. This finding confirms the importance of large tangential vertical structures on the behaviour of the flow. 


\section{CONCLUSION}

Numerical predictions have been performed using a DNS code, the RSM closure of Elena and Schiestel [9] and some LES and RANS models available within OpenFOAM, StarCCM+ or CFX. The computed results have been compared to experimental data available in the literature for an original Couette-Taylor arrangement initially developed by Burin et al. [5] characterized by endcap rings enclosing the cavity.

In the present cavity, the difficulty arises from the presence of two endcap rings attached to both cylinders. It appeared then crucial to take into account accurately the brutal variations in the boundary conditions on the upper and lower walls. A multidomain DNS code has then been developed providing very valuable data for comparisons with the experimental data and other numerical results. DNS showed that there is no evidence of Taylor vortices embedded in the turbulent flow apart very close to the inner cylinder where circular vortices have been observed. It explains why the 2D unsteady RSM [9], which has been sensitized to the implicit effects of rotation on turbulence, provides the best overall agreement in that case, where the rotation rate is quite high. On the contrary, all the k- $\omega$ SST models including the innovative SAS model fail to predict the mean tangential velocity profiles with large discrepancies even in the core of the flow. It can be attributed to the existence of four large recirculations in a meridian plane, whereas all the other models predict only two bubbles. All the models predict the good tendency for the turbulent angular momentum transport $G$, which is found to vary as $R e^{1.9}$ in agreement with previous approaches [7]. Nevertheless, most of the models including the RSM [9], the LES (OpenFOAM) but also the DNS underpredict the shear stress tensor component. The cross correlation coefficient $C_{r \theta}$ is well predicted by all the approaches with almost a constant value around 0.2 (at a given location) for a wide range of Reynolds numbers. The LES provides very useful data showing the high variability of these two parameters.

For industrial applications, the DNS and LES remain computationally expensive. The RSM model [9] showed a good behaviour in this challenging test case providing fair results for the mean and turbulent fields. It offers then a good compromise between accuracy and calculation cost. More comparisons at higher Reynolds numbers are now required to confirm these tendencies.

Acknowledgments: The French part of this work was financially supported by the 
Institut Carnot Star through the TCPtherm project. The postdoctoral position of A. Aubert is supported by Liebherr Aerospace Toulouse. They are here gratefully acknowledged. The authors are also grateful to Dr. Raphaël Guillerm and Prof. Bruno Facchini for their useful suggestions.

[1] S. Abide and S. Viazzo, A 2D compact fourth-order projection decomposition method, J. Comp. Phys., 206, 252-276 (2005).

[2] Y. Bazilevs and I. Akkerman, Large Eddy Simulation of turbulent Taylor-Couette flow using isogeometric analysis and the residual-based variational multiscale method, J. Comp. Phys., 229, 3402-3414 (2010).

[3] F. Beaubert and S. Viazzo, Large Eddy Simulations of plane turbulent impinging jets at moderate Reynolds numbers, Int. J. Heat Fluid Flow, 24 (4), 512-519 (2003).

[4] M. Biage and J.C.C. Campos, Visualization study and quantitative velocity measurements in turbulent Taylor-Couette flow tagging: a description of the transition to turbulence, J. Braz. Soc. Mech. Sci. Eng., 25 (4), 378-390 (2003).

[5] M.J. Burin, E. Schartman and H. Ji, Local measurements of turbulent angular momentum transport in circular Couette flow. Exp. Fluids, 48, 763-769 (2010).

[6] S. Dong, Turbulent flow between counter-rotating concentric cylinders: a direct numerical simulation study, J. Fluid Mech., 615, 371-399, 2008.

[7] B. Dubrulle and F. Hersant, Momentum transport and torque scaling in Taylor-Couette flow from an analogy with turbulent convection, Eur. Phys. J. B, 26, 379-386, 2002.

[8] B. Eckhardt, S. Grossmann and D. Lohse, Torque scaling in turbulent TaylorCouette flow between independently rotating cylinders, J. Fluid Mech., 581, 221-250, 2007.

[9] L. Elena and R. Schiestel, Turbulence modeling of rotating confined flows, Int. J. Heat and Fluid Flow, 17, 283-289 (1996).

[10] N. Fukushima, T. Fushimi, M. Shimura, M. Tanahashi and T. Miyauchi, Dynamics of largeand small-scale vortical structures in turbulent Taylor-Couette flow, 7th Int. Symp. on Turbulence and Shear Flow Phenomena, Ottawa (2007).

[11] R. Guillerm, R. Da Soghe, C. Bianchini, S. Poncet and S. Viazzo, Numerical predictions of flow field in closed and opened Taylor-Couette cavities, 4th European Conference for Aerospace 
Sciences, Saint-Petersburg (2011).

[12] R. Hollerbach and A. Fournier, End-effects in rapidly rotating cylindrical Taylor-Couette flow, AIP Conference proceedings, 733, Ed. Rosner et al., AIP Press, New-York, p.114-121 (2004).

[13] H. Ji, M. Burin, E. Schartman and J. Goodman, Hydrodynamic turbulence cannot transport angular momentum effectively in astrophysical disks, Nature, 444, 343-346 (2006).

[14] A. Kageyama, H. Ji, J. Goodman, F. Chen and E. Shoshan, Numerical and experimental investigation of circulation in short cylinders, J. Phys. Soc. Japan, 73 (9), 2424-2437 (2004).

[15] D. Lathrop, J. Fineberg and H.L. Swinney, Transition to shear-driven turbulence in TaylorCouette flow, Phys. Rev. A, 46, 6390-6408 (1992).

[16] B. E. Launder and D. P. Tselepidakis, Application of a new second moment closure to turbulent channel flow rotating in orthogonal mode, Int. J. Heat Fluid Flow, 15 (1), 2-10 (1994).

[17] G.S. Lewis and H.L. Swinney, Velocity structure functions, scaling, and transitions in high Reynolds number Couette-Taylor flow, Phys. Rev. E, 59 (5), 5457-5467 (1999).

[18] S. Menon, P.K. Yeung and W.W. Kim, Effect of subgrid models on the computed interscale energy transfer in isotropic turbulence, AIAA paper 94-2387 (1994).

[19] F.R. Menter and Y. Egorov, A Scale-Adaptive Simulation Model using Two-Equation Models, AIAA paper 2005-1095, Reno/NV (2005).

[20] F.R. Menter and Y. Egorov, The scale-adaptive simulation method for unsteady flow predictions, Aerodynamic noise from wall-bounded flows, Von Kármán lecture series, 03 (2009).

[21] J. M. Owen, Flow and heat transfer in rotating disc systems, In CHT01 Turbulence Heat and Mass Transfer 3, ed. Y. Nagano, K. Hanjalic, T. Tsuji, 33-58. Tokyo: Aichi Shuppan (2000).

[22] S. Poncet, M. P. Chauve and R. Schiestel, Batchelor versus Stewartson flow structures in a rotor-stator cavity with throughflow, Phys. Fluids, 17, 075110 (2005).

[23] S. Poncet and R. Schiestel, Numerical modeling of heat transfer and fluid flow in rotor-stator cavities with throughflow, Int. J. Heat Mass Transfer, 50 (7-8), 1528-1544 (2007).

[24] S. Poncet, S. Haddadi and S. Viazzo, Numerical modeling of fluid flow and heat transfer in a narrow Taylor-Couette-Poiseuille system, Int. J. Heat Fluid Flow, 32, 128-144 (2011).

[25] G.P. Smith and A.A. Townsend, Turbulent Couette flow between concentric cylinders at large Taylor numbers, J. Fluid Mech., 123, 187-217 (1982).

[26] G.I. Taylor, Stability of a viscous liquid contained between rotating cylinders, Phil. Trans. Roy. Soc. Lond., 223, 289-343 (1923). 
[27] P. Tong, W.J. Goldburg, J.S. Huang and T.A. Witten, Anisotropy in turbulent drag reduction, Phys. Rev. Lett., 65, 2780-2783 (1990).

[28] S. Viazzo, S. Poncet, E. Serre, A. Randriamampianina and P. Bontoux, High-order LES of confined rotor-stator flows, Flow, Turbulence $\&$ Combustion, 88 (1-2), 63-75 (2012).

[29] H.G. Weller, G. Tabor, H. Jasak and C. Fureby, A tensorial approach to computational continuum mechanics using object-oriented techniques, Computers in Physics, 12 (6), 620-631 (1998).

[30] F. Wendt, Turbulente Strömungen zwischen zwei rotierenden konaxialen Zylindern, Ing. Arch., 4, 577-595 (1933).

[31] A. Yoshizawa, Bridging between eddy-viscosity-type and second-order models using a twoscale DIA, 9th Symposium on Turbulent Shear Flows 3: 23.1.1-23.1.6, Kyoto, Japan (1993). 